\title{
Some Locally One-Dimensional Difference Schemes for Parabolic Equations in an Arbitrary Region
}

\author{
By Bert Hubbard
}

1. Introduction. In a paper [6], A. A. Samarskii first proposed a "locally one-dimensional" finite difference scheme for the first boundary problem for a parabolic equation where the cross-section of the cylindrical domain involved was arbitrary. He analyzed the scheme in maximum norm and by means of a discrete form of the maximum principle was able to obtain estimates for the order of convergence. These estimates range from $O(\tau+h)$ to $O\left(\tau+h^{2}\right)$ depending on the nature of the cross-section, cf. Hubbard [4].

In [4] a number of locally one-dimensional schemes are proposed and error analyses made in maximum norm which are either $O(\tau+h)$ or $O\left(\tau+h^{2}\right)$ depending on the particular scheme employed. The point of view taken was to define a finite difference analog at each time level whose matrix was a product of tridiagonal matrices. Such an approach has the merit of allowing a more precise analysis of the contribution to the error from "regular interior" points and "irregular interior" points, i.e. those near the boundary. Such an analysis sheds some light on the difficulties involved in formulating $O\left(\tau^{2}+h^{2}\right)$ schemes for general cross-sections.

In this paper we adopt the point of view of Samarskii [6] and, using his decomposition of the error, we formulate and analyze a series of economical difference schemes for arbitrary cross-sections whose order of convergence ranges from $O(t+h)$ to $O\left(t+h^{2}\right)$ depending on the scheme chosen. The techniques employed in the error analysis are related to those used by Bramble and Hubbard [1] and elaborated in later papers.

Economical difference schemes were first suggested in 1955 by .J. Douglas [2] and Peaceman and Rachford [5] and since then a vast literature has arisen in this area. The reader is referred to the paper of Samarskii [6] and Douglas and Gunn [3] for further references.

2. Locally One-Dimensional Schemes. Since this paper is closely connected with that of Samarskii [6] we shall use notation consistent with his wherever possible. We consider the parabolic equation in $p$ space dimensions

$$
\begin{aligned}
c(x, t) \frac{\partial u}{\partial t} & =L u+f(x, t), \\
L u & =\sum_{\alpha=1}^{p} L_{\alpha} u, \\
L_{\alpha} u & =\frac{\partial}{\partial x_{\alpha}}\left(k_{\alpha}(x, t) \frac{\partial u}{\partial x_{\alpha}}\right)+r_{\alpha}(x, t) \frac{\partial u}{\partial x_{\alpha}}-q_{\alpha}(x, t) u,
\end{aligned}
$$

Received April 15, 1965. This research was supported in part by the National Science Foundation under Grant NSF GP-3666. 
where $x=\left(x_{1}, \cdots, x_{p}\right)$. The functions $c, f, k_{\alpha}, r_{\alpha}$, and $q_{\alpha}$ satisfy certain smoothness criteria which are given in [6]. Let $R$ be a bounded region in $p$ space with boundary $\Gamma$, and

$$
\bar{R}=R \cup \Gamma, \quad \bar{Q}_{T}=\bar{R} \times[0 \leqq t \leqq T], \quad Q_{T}=R \times(0<t \leqq T) .
$$

Let $u(x, t)$ be a solution of

$$
\begin{aligned}
c \frac{\partial u}{\partial t} & =L u+f \quad \text { in } \quad Q_{\boldsymbol{T}}, \\
u_{\mid \mathrm{\Gamma}} & =\mu(x, t) \quad \text { for } \quad x \in \Gamma \quad \text { and, } \quad t \in(0, T], \\
u(x, 0) & =u_{0}(x) \quad \text { for } \quad x \in \bar{R} .
\end{aligned}
$$

We assume further that in $Q_{T}$,

$$
k_{\alpha}(x, t) \geqq k_{*}>0, \quad c(x, t) \geqq c_{*}>0, \quad q_{\alpha}(x, t) \geqq 0 .
$$

The assumption on $q_{\alpha}$ is made only for convenience since a well-known change of dependent variable will cause it to be satisfied. In addition to smoothness assumptions on the data of the problem we require certain smoothness of $u$ as specified in [6].

In the usual manner we place a mesh on the cylinder with mesh constants $h_{\alpha}$, $\alpha=1, \cdots, p$ and $\tau$ in the space and time variables respectively. On any crosssection the mesh points in $R$ make up the set $R_{h}$. The boundary crossings make up the set $\Gamma_{h}$. The "regular" interior points, i.e. those points of $R_{h}$ whose $2 p$ nearest neighbors are in $\bar{R}_{h}=R_{h} \cup \Gamma_{h}$ make up $\omega$, and we define $\omega^{*}=R_{h}-\omega$. Those points of $\omega^{*}$ with a neighbor in the $x_{\alpha}$ direction not in $\bar{R}$ make up the set $\omega_{\alpha}^{*}$. Clearly $\omega^{*}=\mathrm{U}_{\alpha=1}^{p} \omega_{\alpha}^{*}$.

In addition to mesh points at levels $t_{n}, n=1,2, \cdots$ where $t_{n}=n \tau$ we assume that the mesh is further subdivided into the fractional levels $t_{n+\alpha / p}=(n+\alpha / p) \tau$, $\alpha=1, \cdots, p$. With each of the fractional levels we associate the operator $L_{\alpha}$, $\alpha=1, \cdots, p$. It is this operator which we approximate at each of the points of $R_{h}$ by the operator $\Lambda_{\alpha}$. Instead of considering, as does Samarskii, a general class of $O\left(\tau+h_{\alpha}{ }^{2}\right)$ local approximations at points of $R_{h}-\omega_{\alpha}{ }^{*}$, we shall, for the sake of definiteness, take the specific one given by

$$
\Lambda_{\alpha} y \equiv\left(k_{\alpha}\left(x^{\left(-1 / 2_{\alpha}\right)}, t\right) y_{\tilde{x}_{\alpha}}\right)_{x_{\alpha}}+r_{\alpha}(x, t) y_{\dot{x}_{\alpha}}-q_{\alpha}(x, t) y,
$$

where

$$
\begin{gathered}
x^{\left( \pm m_{\alpha}\right)}=\left\{x_{1}, \cdots, x_{\alpha-1}, x_{\alpha} \pm m h, x_{\alpha+1}, \cdots, x_{p}\right\}, \\
y^{\left( \pm m_{\alpha}\right)}=y\left(x^{\left( \pm m_{\alpha}\right)}, t\right) \quad y_{\tilde{x}_{\alpha}}=\frac{y-y^{\left(-1_{\alpha}\right)}}{h_{\alpha}}, \\
y_{x_{\alpha}}=\frac{y^{\left(+1_{\alpha}\right)}-y}{h_{\alpha}}, \quad y_{\dot{x}_{\alpha}}=0.5\left(y_{\dot{x}_{\alpha}}+y_{x_{\alpha}}\right), \\
\sum_{\alpha=1}^{p} r_{\alpha}(x, t)=r(x, t), \quad \sum_{\alpha=1}^{p} q_{\alpha}(x, t)=q(x, t) .
\end{gathered}
$$

In particular, at the level $t_{j+\alpha / p}$, we shall approximate the one-dimensional differential equation

$$
\frac{1}{p} c(x, t) \frac{\partial u}{\partial t}-L_{\alpha} u=f_{\alpha}(x, t)
$$


at points $x \in R_{h}$ by the finite difference equation

$$
\Pi_{\alpha} y \equiv c\left(x, t_{j}^{*}\right) y_{i_{a}}^{j+\alpha / p}-\Lambda_{\alpha} y^{j+\alpha / p}=f\left(x, t_{j}^{*}\right), \quad \sum_{\alpha=1}^{p} f_{\alpha}\left(x, t_{j}^{*}\right)=f\left(x, t_{j}^{*}\right),
$$

where

$$
\begin{aligned}
y^{j+\alpha / p} & \equiv y\left(x, t_{j+\alpha / p}\right), \\
y^{j+\alpha / p} & \equiv \frac{1}{\tau}\left[y\left(x, t_{j+\alpha / p}\right)-y\left(x, t_{j+(\alpha-1) / p}\right)\right]
\end{aligned}
$$

and $t_{j}^{*}$ is a point in the interval $[j \tau,(j+1) \tau]$.

It is easily seen [6] that under suitable smoothness assumptions

$$
\psi_{\alpha}^{*} \equiv \Pi_{\alpha} u-\frac{1}{p} c \frac{\partial u}{\partial t}+L_{\alpha} u=O\left(\tau+h_{\alpha}{ }^{2}\right)
$$

for $x \in R_{h}-\omega_{\alpha}^{*}$, and $t=t_{j}^{*}$ in the last two terms.

At a point $\left(x, t_{j+\alpha / p}\right)$ where $x \in \omega_{\alpha}{ }^{*}$ we define $\Lambda_{\alpha}$ to be the $O\left(\tau+h_{\alpha}\right)$ approximation to $L_{\alpha}$ obtained by interpreting (2.5) in the usual way when the presence of a boundary crossing causes the mesh spacing to be irregular. For example, if $x, x^{+1_{\alpha}} \in R_{h}$ and $x^{-l_{\alpha}} \in \Gamma_{h}$ where $0<l \leqq 1$ then (2.5) becomes

$$
\begin{aligned}
\Lambda_{a} y \equiv & \frac{2}{\left(1+l_{\alpha}\right) h_{\alpha}}\left\{k_{\alpha}\left(x^{\left(1 / 2_{\alpha}\right)}, t\right) y_{x_{\alpha}}-k_{\alpha}\left(x^{\left(-l / 2_{\alpha}\right)}, t\right)\left[\frac{y-y^{\left(-l_{\alpha}\right)}}{l_{\alpha} h_{\alpha}}\right]\right\} \\
& +r_{\alpha}(x, t)\left[\frac{y^{\left(+l_{\alpha}\right)}-y^{\left(-l_{\alpha}\right)}}{\left(1+l_{\alpha}\right) h_{\alpha}}\right]-q_{\alpha}(x, t) y .
\end{aligned}
$$

It is not difficult to establish that under the smoothness assumptions given in [6]

$$
\psi_{\alpha}^{*} \equiv \Pi_{\alpha} u-\frac{1}{p} c \frac{\partial u}{\partial t}+L_{\alpha} u=O\left(\tau+h_{\alpha}\right)
$$

at such a point.

The finite difference analog of (2.1) is now taken to be

$$
\begin{aligned}
\Pi_{\alpha} y & =f_{\alpha}, \quad t=(n+\alpha / p) \tau, \quad x \in R_{h}, \\
y_{\mid \Gamma_{h}} & =\mu, \\
y(x, 0) & =u_{0}(x) .
\end{aligned}
$$

3. Properties of Inverse Matrix. If $\left\{h_{\alpha}\right\}$ are taken to be sufficiently small then the matrix of the system (2.13) is seen to be of positive type [1] and hence the inverse matrix exists and is non-negative. A typical element of the inverse matrix, $g(x, t ; \bar{x}, \bar{t})$, satisfies the equations

$$
\begin{aligned}
\Pi_{\alpha,(x, t)} g(x, t ; \bar{x}, \bar{t}) & =\delta(x, t ; \bar{x}, \bar{t}), & & t=(n+\alpha / p) \tau, \quad x \in R_{h}, \\
g(x, t ; \bar{x}, \bar{t}) & =\delta(x, t ; \bar{x}, \bar{t}), & & x \in \Gamma_{\alpha}, \\
g(x, 0 ; \bar{x}, \bar{t}) & =\delta(x, 0 ; \bar{x}, \bar{t}), & & x \in R_{h},
\end{aligned}
$$

where $\delta$ is the Kronecker delta defined by

$$
\delta(x, t ; \bar{x}, \bar{t})= \begin{cases}1 ; & x=\bar{x} \text { and } t=\bar{t} \\ 0 ; & \text { otherwise. }\end{cases}
$$


In terms of $g$ any mesh function $w(x, t)$ has the representation

$$
\begin{aligned}
w(x, t) \equiv & \sum_{i=\tau / p}^{t} \sum_{\bar{x} \in R_{h}} g(x, t ; \bar{x}, \bar{t}) \tilde{\Pi} w(\bar{x}, \bar{t}) \\
& +\sum_{i=\tau / p}^{t} \sum_{\bar{x} \in \Gamma_{h}} g(x, t ; \bar{x}, \bar{t}) w(\bar{x}, \bar{t})+\sum_{\bar{x} \in R_{h}} g(x, t ; \bar{x}, 0) w(\bar{x}, 0),
\end{aligned}
$$

where $\bar{t}$ in the above sum ranges over $\tau / p, 2 \tau / p, \cdots, \tau,(1+1 / p) \tau, \cdots$, $(n+\beta / p) \tau=t$ and at the level $\bar{t}=(m+\alpha / p) \tau$ the operator $\tilde{\Pi} \equiv \Pi_{\alpha}$ with $m \tau \leqq t^{*} \leqq(m+1) \tau$. As has already been mentioned

$$
g(x, t ; \bar{x}, \bar{t}) \geqq 0 .
$$

We now obtain bounds on certain partial sums of the finite difference Green's function $g(x, t ; \bar{x}, \bar{t})$ which aid in the estimation of the order of convergence.

In (3.3) we set $w(x, t) \equiv 1$ and obtain the inequality

$$
1 \geqq \sum_{i=\tau / p}^{t} \sum_{\bar{x} \in \Gamma_{h}} g(x, t ; \bar{x}, \bar{t})+\sum_{\bar{x} \in R_{h}} g(x, t ; \bar{x}, 0) .
$$

If we set $w(x, t) \equiv$ in (3.3) and use the fact that $\Pi_{\alpha} w \geqq c w_{i_{\alpha}}$ for $x \in R_{h}$ and $w \geqq 0$ on $\Gamma_{h}$ then upon dropping certain non-negative terms it follows that

$$
\frac{p}{c_{*}} \geqq \sum_{i=1 / p}^{t} \sum_{\dot{x} \in R_{h} \omega_{\alpha^{*}}} g(x, t ; \bar{x}, \bar{t}),
$$

where $c_{*}$ is given in (2.4).

Also upon setting

$$
w(x, t)= \begin{cases}1, & x \in R_{h}, \\ 0, & x \in \Gamma_{h},\end{cases}
$$

in (3.3) and noting that $\Pi_{\alpha} w \geqq 0$ for $x \in R_{h}-\omega_{\alpha}{ }^{*}$ and

$$
\Pi_{\alpha} w \geqq \frac{k_{*}}{h_{\alpha}^{2}}\left[\frac{2}{\left(1+l_{\alpha}\right) l_{\alpha}}\right] \geqq \frac{k_{*}}{h^{2}} \quad \text { for } \quad x \in{\omega_{\alpha}}^{*}
$$

then we see that

$$
h^{2} \geqq k_{*} \sum_{i=(n+\alpha / p) \tau=1 / p}^{t} \sum_{\tilde{x} \in \omega_{\alpha^{*}}} g(x, t ; \bar{x}, \bar{t}),
$$

where $k_{*}$ is the lower bound for $k$ given in (2.4) and $h$ is defined by

$$
h^{2}=\sum_{\alpha=1}^{p} h_{\alpha}^{2} .
$$

4. Order of Convergence Estimates. Let the error be denoted by $z$, i.e. $z \equiv u-y$ where $u$ and $y$ are solutions of (2.1) and (2.13) respectively. As does Samarskii [6], we decompose $z$ into

$$
z=v+\eta
$$

where $\eta$ satisfies

$$
\begin{aligned}
c\left(x, t^{*}\right) \eta_{i \alpha} & =\psi_{\alpha}^{0}, \quad x \in \bar{R}_{h}, \quad t=\frac{1}{p}, \cdots, T \\
\eta(x, 0) & =0, \quad x \in \bar{R}_{h},
\end{aligned}
$$


with

$$
\psi_{\alpha}^{0} \equiv \frac{c\left(x, t^{*}\right)}{p} \frac{\partial u}{\partial t}\left(x, t^{*}\right)-L_{\alpha} u\left(x, t^{*}\right)-f_{\alpha}\left(x, t^{*}\right) .
$$

We note that for each level, $m \tau \leqq t^{*} \leqq(m+1) \tau$.

$$
\sum_{\alpha=1}^{p} \psi_{\alpha}^{0}=0
$$

The solution of (4.2), as pointed out by Samarskii, is easily seen to be

$$
\eta\left(x, t_{j+\alpha / p}\right)=(\tau / c) \sum_{\sigma=1}^{\alpha} \psi_{\sigma}{ }^{0}=-(\tau / c) \sum_{\sigma=\alpha+1}^{p} \psi_{\sigma}{ }^{0}=O(\tau) .
$$

We now use the inequalities of Section 3 to obtain estimates for $v$. Clearly for $p \in R_{h}$

$$
\Pi_{\alpha} z=\Pi_{\alpha} u-\Pi_{\alpha} y=\psi_{\alpha}{ }^{0}+\psi_{\alpha}{ }^{*},
$$

where $\psi_{\alpha}{ }^{*}$ is $O\left(\tau+h_{\alpha}\right), p \in \omega_{\alpha}{ }^{*}$ or $O\left(\tau+h_{\alpha}{ }^{2}\right), p \in R_{h}-\omega_{\alpha}{ }^{*}$, and hence

$$
\Pi_{\alpha} v=\Pi_{\alpha} z-\Pi_{\alpha} \eta=\psi_{\alpha}^{*}+\Lambda_{\alpha} \eta
$$

In view of $(2.9),(2.12),(4.5)$ and (4.7) and with proper smoothness assumptions we see that

$$
\pi_{\alpha} v\left(x, t_{j+\alpha / p}\right)= \begin{cases}\frac{1}{c_{*}} O\left(\tau+h_{\alpha}{ }^{2}\right) ; & x \in R_{h}-\omega_{\alpha}{ }^{*}, \\ \frac{1}{c_{*}} O\left(\tau+h_{\alpha}\right) ; & x \in \omega_{\alpha}{ }^{*} .\end{cases}
$$

Substituting $w=v$ into (3.3) and applying inequalities (3.5), (3.6) and (3.8) together with the estimate (4.8) and the fact that $v=-\eta=O(\tau)$ for $x \in \Gamma_{h}$, $v=0$ for $t=0$ we have

$$
\begin{aligned}
|v(x, t)| & \leqq \frac{p}{c_{*}} O\left(\tau+h^{2}\right)+\frac{1}{c_{*} k_{*}} \sum_{\alpha=1}^{p} h_{\alpha}^{2} O\left(\tau+h_{\alpha}\right)+\frac{1}{c^{*}} O(\tau) \\
& =O\left(\tau+h^{2}\right) .
\end{aligned}
$$

In view of (4.5) and (4.9) we see that

$$
z=O\left(\tau+h^{2}\right)
$$

which is the desired error estimate.

5. Other Difference Schemes. As in [4] we shall formulate some alternative locally one-dimensional difference analogs which can be analyzed in the same manner as the one given above. The first of these involves interpolation to the boundary and is somewhat similar to the scheme considered by Samarskii [6]. The two schemes will differ for most regions since the set $\omega_{\alpha}{ }^{*}$ does not normally coincide with the corresponding point set at which Samarskii interpolates to the boundary.

In place of (2.11) we define

$$
\Pi_{\alpha} y \equiv-\Lambda_{\alpha} y \equiv-\left(\frac{2}{\left(1+l_{\alpha}\right) h_{\alpha}}\right)\left\{y_{x_{\alpha}}-\left[\frac{y-y^{\left(-l_{\alpha}\right)}}{l_{\alpha} h_{\alpha}}\right]\right\} \text {. }
$$


The difference analog (2.13) is replaced by

$$
\begin{array}{rlrl}
\Pi_{\alpha} y & =f_{\alpha} ; \quad & x \in R_{h}-\omega_{\alpha}{ }^{*}, \quad t \equiv(n+\alpha / p) \tau, \\
\Pi_{\alpha} y & =0 ; & & x \in \omega_{\alpha}{ }^{*}, \\
y_{\mid \Gamma_{h}} & =\mu, & & \\
y(x, 0) & =u_{0}(x) . & &
\end{array}
$$

It is easily verified that the results of Section 3 again are valid. Equation (4.8) is replaced by

$$
\Pi_{\alpha} v\left(x, t_{j+\alpha / p}\right)= \begin{cases}O\left(\tau+h_{\alpha}{ }^{2}\right) ; & x \in R_{h}-\omega_{\alpha}{ }^{*}, \\ O(1)+O(\tau) ; & x \in \omega_{\alpha}{ }^{*},\end{cases}
$$

where we have suppressed the dependence on $c_{*}$ and $k_{*}$ and consequently (4.9) takes the form

$$
|v(x, t)|=O\left(\tau+h^{2}\right)+h^{2} O(1)=O\left(\tau+h^{2}\right) .
$$

The equation (4.10) is thus seen to be valid in this case.

An $O(\tau+h)$ scheme can be formulated in various ways [4]. We give only one example. Let the sets $R_{h}$ and $\omega^{*}$ be defined as before. Since we wish to look upon $\omega^{*}$ as the "boundary" of our mesh cross-section we define the new point sets $\omega_{\alpha}^{* *}$ to be those points in $R_{h}-\omega^{*}$ with a neighbor in the $x_{\alpha}$ direction which belongs to $\omega^{*}, \alpha=1, \cdots, p$. Our locally one-dimensional scheme is then defined by

$$
\begin{aligned}
\Pi_{\alpha} y & =f_{\alpha} ; \quad x \in R_{h}-\omega^{*}, \quad t=(n+\alpha / p) \tau, \\
y(x, 0) & =u_{0}(x), \\
y(x, t) & =\mu(\bar{x}, t) ; \quad x \in \omega^{*}, \quad \sum_{\alpha=1}^{p}\left(x_{\alpha}-\bar{x}_{\alpha}\right)^{2} \leqq h^{2} .
\end{aligned}
$$

To investigate the error arising from this scheme we develop the inequalities of Section 3 for an "interior" Green's difference function using the following mapping

$$
\begin{aligned}
R_{h} & \rightarrow R_{h}-\omega^{*}, \\
\Gamma_{h} & \rightarrow \omega^{*}, \\
\omega_{\alpha}^{*} & \rightarrow \omega_{\alpha}^{* *} .
\end{aligned}
$$

Using the same mapping we see that the results of Section 4 hold without change through (4.7). Equation (4.8) is replaced by

$$
\Pi_{\alpha} v\left(x, t_{j+\alpha / p}\right)=O\left(\tau+h_{\alpha}^{2}\right) ; \quad x \in R_{h}-\omega^{*} .
$$

Now upon substituting $w=v$ into (3.3) and applying inequalities (3.6) and (3.8) (noting that $\eta=O(\tau)$ and hence $v=O(\tau)+O(h)$, on $\omega^{*}$ ) together with the estimate (5.7) we see that

$$
|v(x, t)|=O\left(\tau+h^{2}\right)+O(\tau)+O(h)=O(\tau+h) .
$$


Consequently in this case we have

$$
z=O(\tau+h) .
$$

University of Maryland

College Park, Maryland

1. J. Bramble \& B. HubBard, "On the formulation of finite difference analogues of the Dirichlet problem for Poisson's equation," Numer. Math., v. 4, 1962, pp. 313-327. MR 26 *7157.

2. J. Douglas, "On the numerical integration of $U_{x x}+U_{y y}=U_{t}$ by implicit methods," J. Soc. Indust. Appl. Math., v. 3, 1955, pp. 42-65.

3. J. Douglas \& J. GưN, "A general formulation of alternating direction methods." (To appear.)

4. B. HUBBARD, "Alternating direction schemes for the heat equation in a general domain." (To appear.)

5. D.W. PEACEMAN, \& H. H. RACHFoRD, "The numerical solution of parabolic and elliptic differential equations,"'J. Soc. Indust. Appl. Math., v. 3, 1955, pp. 28-41. MR 17, 196.

6. A. A. Samarskir, "On an economical difference method for the solution of a multidimensional parabolic equation in an arbitrary region," Ž. Vyčisl. Mat. i Mat. Fiz., v. 2, 1962, pp. 787-811 (Russian); English transl., U.S.S.R. Comput. Math. and Math. Phys., v. 2, 1962, pp. 894-926. 